Harald Pleiner and Helmut R. Brand*

\title{
21 Symmetry aspects in the macroscopic dynamics of magnetorheological gels and general liquid crystalline magnetic elastomers
}

\begin{abstract}
We investigate theoretically the macroscopic dynamics of various types of ordered magnetic fluid, gel, and elastomeric phases. We take a symmetry point of view and emphasize its importance for a macroscopic description. The interactions and couplings among the relevant variables are based on their individual symmetry behavior, irrespective of the detailed nature of the microscopic interactions involved. Concerning the variables we discriminate between conserved variables related to a local conservation law, symmetry variables describing a (spontaneously) broken continuous symmetry (e.g., due to a preferred direction) and slowly relaxing ones that arise from special conditions of the system are considered. Among the relevant symmetries, we consider the behavior under spatial rotations (e.g., discriminating scalars, vectors or tensors), under spatial inversion (discriminating e.g., polar and axial vectors), and under time reversal symmetry (discriminating e.g., velocities from polarizations, or electric fields from magnetic ones). Those symmetries are crucial not only to find the possible cross-couplings correctly but also to get a description of the macroscopic dynamics that is compatible with thermodynamics. In particular, time reversal symmetry is decisive to get the second law of thermodynamics right. We discuss (conventional quadrupolar) nematic order, polar order, active polar order, as well as ferromagnetic order and tetrahedral (octupolar) order. In a second step, we show some of the consequences of the symmetry properties for the various systems that we have worked on within the SPP1681, including magnetic nematic (and cholesteric) elastomers, ferromagnetic nematics (also with tetrahedral order), ferromagnetic elastomers with tetrahedral order, gels and elastomers with polar or active polar order, and finally magnetorheological fluids and gels in a one- and two-fluid description.
\end{abstract}

Keywords: dissipation function, ferromagnetic nematics, hydrodynamics, linear irreversible thermodynamics, magnetorheological fluids, reversible dynamics

\footnotetext{
*Corresponding author: Helmut R. Brand, Department of Physics, University of Bayreuth, Bayreuth, Germany, e-mail: brand@uni-bayreuth.de Harald Pleiner, Max Planck Institute for Polymer Research, Mainz, Germany, e-mail: pleiner@mpipmainz.mpg.de

כ Open Access. (๑) 2020 Harald Pleiner and Helmut R. Brand, published by De Gruyter. (c))BY-NC-ND This work is licensed under the Creative Commons Attribution-NonCommercial-NoDerivatives 4.0 International License. 


\subsection{General aspects of macroscopic dynamics}

\subsubsection{Macroscopic variables}

In a microscopic dynamic description, all (or a very large number of) microscopic degrees of freedom are taken into account. Macroscopic dynamics makes use of only those rather few dynamic variables that are not relaxed to their equilibrium values on long, macroscopic time scales. They come in three classes: (A) conserved quantities that can only be transported and (B) symmetry variables, describing a spontaneously broken continuous symmetry, e.g., a preferred direction that breaks orientational symmetry, but whose orientation is arbitrary, cannot relax at all in the homogeneous limit. Class A and B are the true hydrodynamic variables since for any excitation with frequency $\omega$ and wave vector $k$, there is $\omega \rightarrow 0$ for $k \rightarrow 0$. Class (C) macroscopic variables comprise all quantities that only slowly relax and therefore interact with the hydrodynamic variables on macroscopic time scales (where the many microscopic degrees of freedom have already relaxed to their equilibrium values). There are no general criteria to identify such macroscopic variables, and their existence depends on the nature of the individual system.

Examples for conserved quantities are the mass density $\rho$ (or individual mass densities in a mixture without chemical reactions), the momentum density $g_{i}$, and the energy density $\varepsilon$. Among the symmetry variables, we consider the nematic director $n_{i}$, the vector $p_{i}$ describing the orientation of a polar preferred direction, and $m_{i}$ denoting the direction of a spontaneous magnetization. In active systems with polar order, e.g., moving bird flocks or fish schools, the preferred direction is given by the orientation $f_{i}$ of an active nonzero velocity. In solid (elastic) media, translational symmetry is broken and characterized by the displacement vector $u_{i}$. Macroscopic variables are the magnetization $M_{i}$ in a magnetizable medium, relative velocities $w_{i}$ in a two-fluid system (different constituents moving with two different velocities), and relative rotations $\Omega_{i}$ among different preferred directions or between a preferred direction and rotations of an elastic body. In the framework of SPP1681 we have worked on many of these systems, which will be discussed in detail, in the following [1-15].

Throughout this chapter, we use the notation $m_{i}, g_{i}, n_{i}$ etc., to characterize vectorial quantities, with indices $i$ running from 1 to 3 in three dimensions. This is a standard notation used in physical hydrodynamics [16].

The variables described above are space-time fields, e.g., $\varepsilon(\boldsymbol{r}, t)$, that live on macroscopic time and length scales. In the Eulerian description, they are volume densities (of the total quantities of the whole system). The basic assumption to derive general equations for those variables is the applicability of thermodynamics. We start with the local formulation of the first law of thermodynamics, which can be interpreted as energy balance of the system. It relates changes of all the variables discussed above to changes of the entropy density $d \sigma$ 


$$
d \varepsilon=T d \sigma+\mu_{A} d A+\psi_{k}^{B} d \nabla_{k} B+\mu_{C} d C
$$

with $T$ the temperature. The prefactors of the differentials are called (thermodynamic) conjugates.

The energy density is assumed to be a scalar, meaning it does not change under rotations. Thus, all the terms added up in Eq. (21.1) must be scalars. This is trivially fulfilled for $T d \sigma$ and for $\mu d \rho$, where $\mu_{A} d A \rightarrow \mu d \rho$ with $\mu$ the chemical potential. If $A$ is a vector, like the momentum density, also $\mu_{A}$ has to be a vector connected by the scalar product, $\mu_{A} d A \rightarrow v_{i} d g_{i}$ with $v_{i}$ the velocity. The $B$ variables must not change the energy, so their contribution in Eq. (21.1) already contains a gradient. As a consequence also their conjugates must be vectors. Generally the $B$ variables are vector-like quantities by themselves, e.g., $B \rightarrow n_{i}$, with $\psi_{k}^{B} d \nabla_{k} B \rightarrow \psi_{i k}^{n} d \nabla_{k} n_{i}$. In the case of the nematic director, external (electric or magnetic) fields are important and an additional contribution is added, $h_{i}^{n I} d n_{i}$, where $h_{i}^{n I}$ is due to the dielectric or diamagnetic anisotropy of the nematic phase. Often the two contributions are combined into $h_{i}^{n} d n_{i}$, with $h_{i}^{n}=$ $h_{i}^{n I}-\nabla_{k} \psi_{i k}^{n}$ and $n_{i}$ belonging to class $C$.

Similarly, when the gradient of the displacement vector $\nabla_{k} u_{i}$ is replaced by a symmetric second-rank strain tensor $U_{i j}$, eliminating solid body rotations that must not contribute to the energy density, $U_{i j}$ belongs to class $C$ with $\mu_{C} d C \rightarrow \Phi_{i j} d U_{i j}$, where $\Phi_{i j}$ is the elastic stress tensor. For a thorough discussion of the relation of $u_{i}$ with $U_{i j}$ in the nonlinear case, we refer to the study by Pleiner et al. [17].

For the magnetization $M_{i}$ in a magnetizable system, we have $\mu_{C} d C \rightarrow h_{i}^{M} d M_{i}$ with $h_{i}^{M}$ the internal magnetic field. In a ferromagnetic system, $M_{i}=M m_{i}$ can be split into the unit vector $m_{i}$, denoting the direction of the spontaneous magnetization, which is a class $B$ variable with $\mu_{B} d B \rightarrow h_{i}^{m} d m_{i}$, and into the strength of magnetic order $M$, a class $C$ variable with $\mu_{C} d C \rightarrow h^{M} d M$. For active polar order, $F_{i}=F f_{i}$, the direction $f_{i}$ is a class $B$ variable with $\mu_{B} d B \rightarrow h_{i}^{f} d f_{i}$, while $F$ relaxes to its constant stationary value provided by the active entities of the system and is a class $C$ variable. [18]. Relative rotations are rotations of, e.g., the ferromagnetic direction $\delta m_{i}$ relative to rotations of the elastic media, $2 \Omega_{i j} \equiv \nabla_{i} u_{j}-\nabla_{j} u_{i}$. They are linearly defined by $\Omega_{i}=m_{j} \Omega_{i j}-\delta m_{i}$ and are class $C$ variables with $\mu_{C} d C \rightarrow L_{i}^{\Omega} d \Omega_{i}$. For a nonlinear definition of relative rotations, refer the study by Menzel et al. [19].

The statics of a system is then given by the phenomenological relation between the thermodynamic quantities and the variables. These relations involve static susceptibilities, e.g., compressibility, specific heat, thermal expansion for the scalar variables, Frank-type rank-4 tensors with Frank coefficients for $B$ variables, and the elastic tensor containing elastic moduli or a rank-2 rotational tensor for the $C$ variables. An efficient way of setting up these static relations is to use a phenomenological energy functional of all variables, which is given as follows:

$$
E=\int d V \varepsilon\left(\left\{\sigma, A, \nabla_{k} B, C\right\}\right)
$$


from which the conjugates follow by variational derivation according to Eq. (21.1). The crucial point is that the functional is restricted by symmetries, in particular space inversion and time reversal symmetry.

Before we discuss these symmetries in the following, we give here the general form of the dynamic equations for examples of the different classes of variables:
$\boldsymbol{A}$
$(\partial / \partial t) \rho+\nabla_{k} j_{k}^{\rho}=0$
$\boldsymbol{A} \quad(\partial / \partial t) g_{i}+\nabla_{j} \sigma_{i j}=0$
$\boldsymbol{B} \quad(\partial / \partial t) n_{i}+Y_{i}^{n}=0$
C $\quad(\partial / \partial t) U_{i j}+X_{i j}^{(e l)}=0$
C $\quad(\partial / \partial t) \Omega_{i}+X_{i}^{\Omega}=0$
C $\quad(\partial / \partial t) M_{i}+Y_{i}^{M}=0$

defining the mass current density $j_{k}^{(\rho)}$ and the stress tensor $\sigma_{i j}$ for $A$ variables and the (quasi-) currents $Y_{i}^{n}$ and $X_{i j}^{(e l)}, X_{i}^{(\Omega)}$, and $Y_{i}^{M}$ for the others. The $A$ variables show a divergence in the dynamics, while the $B$ variables come with a gradient in the statics. As an effect, both types of variables give rise to the truly hydrodynamic behavior, $\omega(k \rightarrow 0) \rightarrow 0$, while the $C$ variables do not.

\subsubsection{Spatial inversion symmetry: statics}

In three-dimensional space, not only rotations but also spatial inversion, $S_{I}$ where $\boldsymbol{r} \rightarrow-\boldsymbol{r}$, is an important symmetry operation. Physical quantities behave differently under $S_{I}$, either they are invariant ("symmetric", or "even") with the signature $\epsilon_{S}=+1$ or they change sign (they are "antisymmetric" or "odd") with $\epsilon_{S}=-1$. The signatures $\epsilon_{S}= \pm$ are the only possibilities since when inversion is applied twice, the original state is regained, $S_{I}^{2}=+1$.

(True) scalar quantities have $\epsilon_{S}=+1$, e.g., the variables $\rho, \sigma$, and $\varepsilon$, and also the conjugates $T$ and $\mu$. (True) vectors are odd with $\epsilon_{S}=-1$, in particular, the polarization and the polar preferred direction, electric fields, and the gradient $\nabla_{i}$, as well as the velocity-type quantities like $g_{i}, v_{i}$, but also a relative velocity $w_{i}$. There is a different kind of vectors, called axial vectors, that are even under $S_{I}$ with $\epsilon_{S}=+1$. Among them, there is the vorticity $2 \omega_{i}=\epsilon_{i j k} \nabla_{j} v_{k}$, the magnetization $M_{i}$, and any magnetic field $H_{i}$. Relative rotations $\Omega_{i}$ are also axial vectors. Slightly more complicated is the case of nematic order. Usually the director $n_{i}$ is treated as a vector with the additional constraint that all equations involved are invariant under the replacement $n_{i} \rightarrow-n_{i}$. Obviously, the director cannot be a polar vector and has to be treated as an axial one, as a necessary condition. We note that the $n_{i} \rightarrow-n_{i}$ invariance is a stronger constraint. 
Since the energy density in Eq. (21.1) has $\epsilon_{S}=+1$, the conjugates of $A$ and $C$ class variables must have equal signatures, while for $B$ class variables, the $S_{I}$ behavior of the conjugates is opposite to that of the variables due to the gradient involved. For the director, it means $\psi_{i j}^{n}$ has $\epsilon_{S}=-1$ but $h_{i}^{n}$ has $\epsilon_{S}=+1$. Regarding the dynamics, Eqs. (21.3)-(21.8), the time $t$ is $S_{I}$ symmetric, and therefore, the quasi-currents of $B$ and $C$ class variables have the same spatial signature as the appropriate variables. For $A$ class variables, this is opposite due to the gradient involved.

Depending on the symmetry of a given system, it can be $S_{I}$ symmetric ("centrosymmetric") or not. Prominent examples for the latter are chiral phases, like cholesteric liquid crystals. Such phases show optical activity (rotation of the plane of polarization of light) that is described by a pseudoscalar quantity $q_{0}$ with $\epsilon_{S}=-1$. The use of $q_{0}$ in the macroscopic dynamic equations exactly characterizes those contributions and couplings that are specific for the chiral system and that would be forbidden in a achiral phase without a $q_{0}$. Well-known examples are the linear twist term in the Frank energy in cholesterics that give rise to the helical ground state and the static couplings between director rotations and, e.g., the thermal degree of freedom leading to the static Lehmann effect [20], absent in the achiral nematic phase. The origin of the existence of a $q_{0}$ can be microscopic, when chiral molecules are present, or it is a result of a complicated internal structure like in the smectic $C_{B 2}$ phase [21]. In the latter case, $q_{0}$ and $-q_{0}$ lead to two chiral structures with opposite handedness but with the same energy for the ground state: ambidextrous chirality [22].

The existence of a polar vector $p_{0}$ also leads to a noncentrosymmetric phase, like polar nematics $[23,24]$. Again, the occurrence of $p_{0}$ denotes those contributions that would not be allowed in a centrosymmetric phase. Such a phase is not chiral.

A third possibility to break $S_{I}$ symmetry in a phase is due to the existence of tetrahedral (or octupolar) order described by a third-rank tensor order parameter $T_{i j}=\sum_{\alpha=1}^{4} n_{i}^{(\alpha)} n_{j}^{(\alpha)} n_{k}^{(\alpha)}$, where the $n_{i}^{(\alpha)}$ are the position vectors of the corners of a tetrahedron [25]. $T_{i j k}$ has $\epsilon_{S}=-1$ since the $n_{i}^{(\alpha)}$ are true vectors. The hydrodynamic variables related to tetrahedral order are the 3-D rigid rotations $d \Gamma_{i} \sim \epsilon_{i p q} T_{p k l} d T_{q k l}$ of the $T_{i j k}$ structure. This is a class $B$ variable with $\psi_{k}^{B} d \nabla_{k} B \rightarrow \psi_{i k}^{\Gamma} d \nabla_{k} \Gamma_{i}$ and

$$
(\partial / \partial t) \Gamma_{i}+Y_{i}^{\Gamma}=0
$$

The variable $\Gamma_{i}$ is $S_{I}$ symmetric as is the quasi-current $Y_{\mathrm{i}}^{\Gamma}$, while $\psi_{i k}^{\Gamma}$ is odd. Similar to the nematic case, one can introduce a $h_{i}^{\Gamma}$ that contains $\nabla_{j} \psi_{i j}^{\Gamma}$ and is even under $S_{I}$. There are subtleties in the nonlinear regime due to the non-Abelian nature of three-dimensional rotations, for details refer the study by Brand and Pleiner [26]. A phase that only contains tetrahedral order is neither chiral nor polar. If there is additionally nematic order (along a 2-fold symmetry axis of the tetrahedron), a $\mathrm{D}_{2 d}$ phase occurs that is still not chiral (and not polar) but shows ambidextrous helicity [27]. Only when the tetrahedral structure is suitably combined with a biaxial nematic order, a chiral (but still 
nonpolar) phase $\mathrm{D}_{2}$ can occur, where the $q_{0}$ is due to the different preferred directions involved [28]. Again, this is an example for ambidextrous chirality.

\subsubsection{Time reversal symmetry}

Under time reversal $T_{R}$, where $t \rightarrow-t$, all macroscopic quantities are either even $\left(T_{R}\right.$ symmetric) with $\epsilon_{T}=+1$ or they are odd ( $T_{R}$ antisymmetric) with $\epsilon_{T}=-1$ since $T_{R}^{2}=+1$. There is no need to discriminate among the $A, B, C$ case variables because the gradient is $T_{R}$ symmetric, $\epsilon_{T}\left(\nabla_{i}\right)=+1$. The energy density is $T_{R}$ symmetric, and therefore, the conjugates have the same $T_{R}$ signature as the variables, $\epsilon_{T}(e)=\epsilon_{T}\left(C_{e}\right)$ for any variable $e$ with conjugate $C_{e}$. Examples for $T_{R}$ symmetric variables are $\rho, \sigma, n_{i}, p_{i}, U_{i j}$, and $\Omega_{i}$, as well as their conjugates $\mu, T, h_{i}^{n}, h_{i}^{p}, \Phi_{i j}$, and $L_{i}^{\Omega}$. Odd quantities with $\epsilon_{T}=-1$ are $g_{i}$ and all types of velocities, including relative velocities and vorticity, $M_{i}$, and $h_{i}^{M}$, as well as magnetic fields.

$T_{R}$ is closely related to the second law of thermodynamics. The latter states that reversible, time reversal symmetric processes with $\epsilon_{T}=+1$ must not change the entropy - they are nondissipative, while irreversible processes with $\epsilon_{T}=-1$ must increase the entropy - they are dissipative. Therefore, one can uniquely split up any current or quasi-current into a reversible (superscript $R$ ) and an irreversible part (superscript $D$ ), e.g., for a variable $e$, the following equation can be written:

$$
(\partial e / \partial t)+Y_{e}^{R}+Y_{e}^{D}=0 .
$$

If time is reversed, Eq. (21.10) reads $(\partial e / \partial t)+Y_{e}^{R}-Y_{e}^{D}=0$. independent of the signature $\epsilon_{T}(e)$.

Thus, $\epsilon_{T}\left(Y_{e}^{R}\right)=\epsilon_{T}(e)$ and $\epsilon_{T}\left(Y_{e}^{D}\right)=-\epsilon_{T}(e)$. In particular,

$$
\begin{gathered}
\epsilon_{T}\left(Y_{e}^{R}\right)=\epsilon_{T}(\partial e / \partial t)= \begin{cases}-1 & \text { for } T_{R}-\text { even } e \\
+1 & \text { for } T_{R}-\text { odd } e\end{cases} \\
\epsilon_{T}\left(Y_{e}^{D}\right)=-\epsilon_{T}(\partial e / \partial t)= \begin{cases}+1 & \text { for } T_{R}-\text { even } e \\
-1 & \text { for } T_{R}-\text { odd } e\end{cases}
\end{gathered}
$$

Among the reversible currents, $\sigma_{i j}^{R}, Y_{i}^{M R}$ are $T_{R}$ even with $\epsilon_{T}=+1$, while $j_{i}^{\rho R}$, $Y_{i}^{n R}$, and $X_{i j}^{(e l) R}$ are odd, with $\epsilon_{T}=-1$. On the other hand, the dissipative currents $\sigma_{i j}^{D}, Y_{i}^{M D}$ have $\epsilon_{T}=-1$, while $j_{i}^{\rho D}, Y_{i}^{n D}$, and $X_{i j}^{(e l) D}$ are even, $\epsilon_{T}=+1$.

We add the entropy balance equation (which is a conservation law in the reversible case) and the energy density conservation law to obtain the following equations:

$$
\begin{gathered}
\partial \sigma / \partial t+j_{i}^{\sigma}=2 R / T \\
\partial \varepsilon / \partial t+j_{i}^{\varepsilon}=0
\end{gathered}
$$


with the dissipation function $R$ as the entropy source term. Putting all macroscopic dynamic equations, including Eqs. (21.3)-(21.8), into the Gibbs relation, Eq. (21.1), one gets a representation of the dissipation function in terms of the dissipative currents and thermodynamic forces

$$
\begin{aligned}
& 2 R=-j_{i}^{\sigma D} \nabla_{i} T-\sigma_{i j}^{D} A_{i j}+Y_{i}^{n D} h_{i}^{n}+Y_{i}^{M D} h_{i}^{M} \\
& +X_{i j}^{(e l) D} \Phi_{i j}+X_{i}^{\Omega D} L_{i}^{\Omega}+Y_{i}^{\Gamma D} h_{i}^{\Gamma}>0
\end{aligned}
$$

Note that for case $C$ variables, the thermodynamic forces are just the conjugates, while for case $A$ variables (the conserved variables), the forces are the gradients of the conjugates $2 A_{i j}=\nabla_{j} v_{i}+\nabla_{i} v_{j}$. Case $B$ variables (symmetry variables) have been written in the form of class $C$ variables, e.g., with $h_{i}^{n}$, the conjugate to $d n_{i}$, as force, rather than using $\psi_{i j}^{n}$, the conjugate to $d \nabla_{j} n_{i}$. There is no dissipative contribution $\sim j_{i}^{\rho D}$ since the mass density current is the momentum density $\rho v_{i}=g_{i}$, which is reversible.

In Eq. (21.15), pure divergence terms have been omitted since they do not contribute to the total entropy production $\sim \int R d V$ in the bulk. $R$ is even under $T_{R}$ since in the opposite case, one would get $R<0$ for time reversed dissipative processes in contradiction to thermodynamics.

The dissipation function $R$ can be used as a potential for dissipative currents. Within linear irreversible thermodynamics, with a linear relationship between currents and forces, one can set up phenomenologically $R$ as a harmonic function of the thermodynamic forces. The dissipative currents then follow by partial derivation of $R$ with respect to the forces. Examples are $j_{i}^{\sigma D}=-\partial R / \partial \nabla_{i} T, Y_{i}^{n D}=\partial R / \partial h_{i}^{n}$, and $X_{i}^{(e l) D}=\partial R / \partial \Phi_{i j}$. If $R$ contains nonvanishing $\Phi_{i j}$ contributions, e.g., $\sim \Phi_{i j} \Phi_{k l}$, the resulting $X_{i j}^{(e l) D}$ describes relaxation of the strain tensor. This is the hydrodynamic description of viscoelasticity of non-Newtonian fluids or relaxing gels. In solids or chemically bonded elastomers, only gradients of the elastic stresses act as forces, e.g., $R \sim\left(\nabla_{j} \Phi_{i j}\right)\left(\nabla_{l} \Phi_{k l}\right)$, and the strains behave as conserved quantities ("permanent elasticity") showing diffusion rather than relaxation.

Generally, the dissipative currents are related to the forces by linear relations

$$
Y_{a}^{D}=\zeta_{a b}^{D} F_{b}
$$

where $a$ and $b$ denote the variables involved. By the very existence of the potential $R, \zeta_{a b}^{D}$ is symmetric, $\zeta_{a b}^{D}=\zeta_{b a}^{D}$. Of course, such relations have to fulfill the $T_{R}$ symmetry properties discussed above, in particular, $\epsilon_{T}\left(\zeta_{a b}^{D}\right)=\epsilon_{T}(a b)$. If the (dissipative) transport parameter $\zeta_{a b}^{D}$ is constant or only depends on structural properties that are $T_{R}$ symmetric, like the nematic director, only variables of equal $T_{R}$ signature can couple, $\epsilon_{T}(a)=\epsilon_{T}(b)$. On the other hand, if there is a $T_{R^{-}}$odd quantity present, like the magnetization or a magnetic field, an odd power of them in $\zeta_{a b}^{D}$ leads to $\epsilon_{T}\left(\zeta_{a b}^{D}\right)=-1$, and the variables $a$ and $b$ must behave oppositely $\epsilon_{T}(a)=-\epsilon_{T}(b)$. 
For the reversible dynamics, there are somewhat different rules. Since the reversible currents must not contribute to $R$, they have to fulfill the following equation:

$$
\begin{aligned}
0= & -j_{i}^{\rho R} \nabla_{i} \mu-j_{i}^{\sigma R} \nabla_{i} T-\sigma_{i j}^{R} A_{i j}+Y_{i}^{n R} h_{i}^{n} \\
& +Y_{i}^{M R} h_{i}^{M}+X_{i j}^{(e l) R} \Phi_{i j}+X_{i}^{\Omega R} L_{i}^{\Omega}
\end{aligned}
$$

replacing Eq. (21.15) for the dissipative currents. Since the bilinear products of thermodynamic forces and reversible currents add up to zero, there is no potential, from which the latter can be derived.

The reversible currents come in two parts, $Y_{a}^{R}=Y_{a}^{0}+\tilde{Y}_{a}^{R}$, where $Y_{a}^{0}$ are the nonphenomenological, material-independent, symmetry-given contributions, and $\tilde{Y}_{a}^{R}$ are the phenomenological parts characterized by (reversible) transport coefficients. The former comprise advection, convection, and rotational covariant derivatives, in addition to, e.g., the isotropic pressure $p$ and the Ericksen stress in the stress tensor, and read as follows [2, 16, 29]:

$$
\begin{gathered}
j_{i}^{\rho 0}=\rho v_{i} \\
j_{i}^{\sigma 0}=\sigma v_{i} \\
j_{i}^{\varepsilon 0}=(\varepsilon+p) v_{i} \\
\sigma_{i j}^{0}=g_{i} v_{j}+\delta_{i j} p-\Phi_{i j}+\Phi_{j k} U_{i k}+\Phi_{i k} U_{j k} \\
+\frac{1}{2}\left(\psi_{k i}^{n} \nabla_{j} n_{k}+\psi_{k j}^{n} \nabla_{i} n_{k}\right) \\
Y_{i}^{n 0}=v_{k} \nabla_{k} n_{i}+\epsilon_{i j k} n_{j} \omega_{k} \\
Y_{i}^{M 0}=v_{k} \nabla_{k} M_{i}+\epsilon_{i j k} M_{j} \omega_{k} \\
X_{i j}^{(e l) 0}=v_{k} \nabla_{k} U_{i j}-A_{i j}+U_{k j} \nabla_{i} v_{k}+U_{k i} \nabla_{j} v_{k} .
\end{gathered}
$$

with $p=-\varepsilon+\mu \rho+T \sigma+v_{i} g_{i}+h_{i}^{M} M_{i}$ containing all extensive variables and their conjugates. The stress tensor has been symmetrized in order to guarantee angular momentum conservation using the condition that Eq. (21.1) is invariant under rotations and that a divergence of an antisymmetric term is irrelevant [16]. All nonphenomenological contributions together fulfill Eq. (21.17).

Concerning the phenomenological parts, we first note that $\tilde{j}_{i}^{\rho R}=0$ because $(\partial \rho) /(\partial t)+\nabla_{i} g_{i}=0$. For the other currents, one has to set up the linear current-force relations explicitly

$$
\tilde{Y}_{a}^{R}=\zeta_{a b}^{R} F_{b}
$$


In accordance with Eq. (21.17), there must be a counter term $\tilde{Y}_{a}^{R}=\zeta_{a b}^{R} F_{a}$ such that $\zeta_{a b}^{R}+\zeta_{b a}^{R}=0$ indicating antisymmetry with respect to $a$ and $b$. Of course, the relation Eq. (21.25) has to be invariant under $T_{R}$ with the result $\epsilon_{T}\left(\zeta_{a b}^{R}\right)=\epsilon_{T}\left(\tilde{Y}_{a}^{R} F_{b}\right)$ or $\epsilon_{T}\left(\zeta_{a b}^{R}\right)=$ $-\epsilon_{T}(a b)$ due to $\epsilon_{T}\left(F_{b}\right)=\epsilon_{T}\left(C_{b}\right)=\epsilon_{T}(b)$. If $\zeta_{a b}^{R}$ is constant or only depends on structural properties that are $T_{R}$ symmetric, like the nematic director, only variables of opposite $T_{R}$ signature can couple, $\epsilon_{T}(a)=-\epsilon_{T}(b)$. On the other hand, if there is a $T_{R}$-odd quantity present, like the magnetization or a magnetic field, an odd power in $\zeta_{a b}^{R}$ leads to $\epsilon_{T}\left(\zeta_{a b}^{R}\right)=-1$, and variables $a$ and $b$ of the same $T_{R}$ signature can couple. In such systems, it is possible to have dissipative as well as reversible self-couplings (or crosscouplings between two given variables), when the dissipative and reversible transport parameters have different $T_{R}$ signatures. An example is (isotropic) heat conduction in ferromagnetic systems, where $j_{i}^{\sigma R}=-\kappa \nabla_{i} T$ and $\tilde{j}_{i}^{\sigma R}=-\kappa^{T} \epsilon_{i j k M_{k}} \nabla_{j} T$ [12]; we note that the latter contribution also exists when linearized since in a ferromagnetic system $\left\langle M_{i}\right\rangle \neq 0$.

Time reversal symmetry is often overlooked and sometimes mistreated as shown in the study by Brand et al. [1, 11].

\subsubsection{Spatial inversion symmetry: dynamics}

Of course, spatial inversion $S_{I}$ is relevant for the dynamics as well. From Eqs. (21.3)-(21.8), we deduce that the physical currents of class $A$ variables (e.g., $j_{i}^{\rho}$ and $\sigma_{i j}$ ) have an $S_{I}$ signature opposite to that of the variables, $\epsilon_{S}\left(j_{a}\right)=-\epsilon_{S}(a)$, while for all other variables, the quasi-currents have $\epsilon_{S}\left(Y_{a}\right)=+\epsilon_{S}(a)$. This applies to the reversible parts, as well as well as to the irreversible ones. Writing the second law of thermodynamics in the form of Eqs. (21.15) and (21.17), the forces for class $A$ variables are gradients of the conjugates (defined in Eq. (21.1)) with the result $\epsilon_{S}\left(F_{a}\right)=$ $-\epsilon_{S}\left(C_{a}\right)=-\epsilon_{S}(a)$, while for the other variables, the forces are just the conjugates resulting in $\epsilon_{S}\left(F_{a}\right)=\epsilon_{S}\left(C_{a}\right)=\epsilon_{S}(a)$.

For the dissipative and reversible transport parameters $\zeta_{a b}^{(D, R)}$ defined in Eqs. (21.16) and (21.25), this implies $\epsilon_{S}\left(\zeta_{a b}\right)=+\epsilon_{S}(a b)$ for all types of variables. Thus, if $\zeta_{a b}$ is a constant or only depends on structural properties that are $S_{I}$ symmetric, like the nematic director, only variables of equal $S_{I}$ signature can couple, $\epsilon_{S}(a)=+\epsilon_{S}(b)$. On the other hand, if there is a $S_{I^{-}}$odd quantity present, like the pseudoscalar $q_{\mathrm{o}}$ or the tetrahedral order $T_{i j k}$, an odd power of those in $\zeta_{a b}$ leads to $\epsilon_{S}\left(\zeta_{a b}\right)=-1$, and the variables $a$ and $b$ must behave oppositely $\epsilon_{S}(a)=-\epsilon_{S}(b)$. Examples are the dissipative dynamic Lehmann effect in chiral systems, where, e.g., temperature gradients (polar vectors) can couple to director rotations (axial vectors) $[11,30]$ and flow-induced reversible (heat) fluxes in tetrahedral systems, where the nonpolar $A_{i j}$ couples to the polar vector $j_{i}^{\sigma R}$ [31]. 


\subsection{Nematic order and magnetism}

\subsubsection{Isotropic and uniaxial magnetic elastomers and gels}

Isotropic ferrogels are systems without a preferred direction combining the properties of a gel and of a conventional magnetic liquid. The hydrodynamic description of isotropic ferrogels has been presented in the study by Jarkova et al. [32]. Quite recently, a continuum model for ferrogels from an engineering perspective has been developed [33]. In parallel microscopic studies of the field-controlled change of shape and elasticity of magnetic gels using particle-based simulations have been advanced; these developments have been elucidated in the study by Weeber et al. [34]. A large effort has been dedicated to the mesoscopic characterization of magnetic hybrid materials such as magnetic gels and elastomers over the last few years, compare, for example, the study by Menzel [35] for a recent review. Important progress along these lines has been achieved for the tunable dynamic moduli of magnetic elastomers on the mesoscale by combining experimental data from X-ray tomography with coarse-grained dipole spring modeling [36]. From an applied point of view, there has been a special emphasis on the magnetic field-controlled mechanical behavior of magnetosensitive elastomers in applications for actuator and sensor systems [37].

Uniaxial magnetic gels with a permanent magnetic moment have been synthesized by performing the cross-linking process in an external magnetic field [38] and afterward characterized with respect to their optical, magnetic, and mechanical properties [38]. For the hydrodynamic description of uniaxial magnetic gels and elastomers, we refer to the study by Bohlius et al. [39]. In the study by Menzel [40], it has been demonstrated how one can bridge the scales from particles to macroscopic length scales in a uniaxial magnetic gel. Very recently, the structure and the magnetooptical response of anisotropic fibrillous organoferrogels with mobile magnetic nanoparticles have been investigated [41]. While in this study no magnetic hysteresis has been found but an optical hysteresis is detected, revealing a complex interplay between the gel and the mobile magnetic particles [41].

\subsubsection{Magnetic nematic elastomers and gels}

Nematic elastomers with a magnetic degree of freedom are described by the director, the strain field, relative rotations between director and elastic rotations, and by a magnetization variable that is zero in equilibrium [2]. We concentrate here on the magnetic effects. There are reversible dynamic couplings between director rotations and the magnetization dynamics, $\left(\partial n_{i} / \partial t\right) \sim h_{j}^{M}$ and $\left(\partial M_{i} / \partial t\right) \sim h_{j}^{n}$, and similarly between relative rotations and the magnetization, $\left(\partial \Omega_{i} / \partial t\right) \sim h_{j}^{M}$ and $\left(\partial M_{i} / \partial t\right) \sim L_{j}^{\Omega}$, all with reversible material tensors of the form $\sim \epsilon_{i j k} n_{k}$. The symbol $\sim$ in the relations above 
indicates that the vectorial quantities on the left and on the right hand side are connected by second-rank tensors. It is the different $T_{R}$ signature of the variables involved and the antisymmetry of the material tensors that allow these couplings. Applying an external magnetic field, rotations of the director and relative rotations are induced (through $h_{i}^{M}$ ). Through similar reversible couplings, external shear flow induces relative rotations.

In ordinary nematics flow alignment of the director is a prominent feature: shear flow rotates the director at a finite angle w.r.t. the flow direction. This angle is independent of the shear rate $\Sigma$ and depends on a reversible flow parameter. In the present case, shear flow also induces relative rotations, which induce a magnetization, and finally, all three variables, $n_{i}, \Omega_{i}$, and $M_{i}$ are oriented, independent of the shear rate. Only the magnitude of the magnetization and the relative rotations are proportional to $\Sigma$. Since the system is elastic, the shear rate has to be oscillatory, $\Sigma \cos \left(\omega_{s} t\right)$. Since there is static coupling between relative rotations and strains, the relative rotations show a phase shift compared to the shear rate indicating that in this complicated system, flow alignment is no longer a pure reversible feature.

If a chiral agent is added or a chiral nematogenic molecule is used, a chiral magnetic nematic elastomer or gel is obtained [3]. The only new structural element compared to the achiral case is the pseudoscalar $q_{0}$ that is odd under $S_{I}$. A well-known consequence is the occurrence of a linear (nematic) twist term in the energy density functional, giving rise to a helical ground state, as well as bilinear coupling terms between twist and temperature and density changes and strains. The latter describe the temperature, density, and strain dependence of the helical pitch. They also give rise to the static part of the Lehmann effect. Note that there are no static couplings to changes of the magnetization due to the odd $T_{R}$ signature of the latter.

In the dynamics, the presence of $q_{\mathrm{O}}$ allows couplings between polar currents and axial forces (and vice versa) like, e.g., $j_{i}^{\sigma R} \sim q_{0} h_{j}^{M}$ and $Y_{i}^{M R} \sim q_{0} \nabla_{j} T$. Applying a temperature gradient along the helical axis, $\nabla_{z} T$, the growth of $M_{z}$ is induced that saturates into a finite stationary magnetization, $M_{z}^{(\text {stat })} \sim q_{0} \nabla_{z} T$. Instead of the temperature gradient, also an external electrical field can be used, thus creating an electric fieldinduced magnetization [3]. The elastic degree of freedom is irrelevant for these effects.

\subsubsection{Ferromagnetic nematics}

Ferromagnetic nematic liquid crystals are fluids with two different kinds of internal order, a nematic one due to the ordering of standard nematogenic molecules and a ferromagnetic one due to the ordering of magnetic nanoplatelets made of complicated iron oxide compounds. Such suspensions have been realized by Mertelj et al. [42, 43]. In equilibrium, the two preferred directions $n_{i}$ and $m_{i}$ are parallel. Thus, there is an energetic penalty, $A_{2}\left[(n \cdot m)^{2}-1\right]$, for deviations from the ground state. In addition, there 
are various (reversible and irreversible) dynamic couplings of these two variables. The full hydrodynamic description for ferromagnetic nematics has been given in the study by Potisk et al. [9], which is a generalization of an earlier work [44] on "ferronematics", i.e., magnetic nematic liquid crystals without permanent magnetization. From a microscopic point of view, the study of the structure and rheology of hybrid mixtures of magnetic nanoparticles in liquid crystals using particle resolved simulations has started a few years ago. Quite recently, the focus in this area has been on the transport properties in liquid crystal-magnetic colloid mixtures [45].

A particularly interesting cross-coupling is the (simplified) dissipative dynamics $[8,44]$

$$
\begin{aligned}
& Y_{i}^{n D}=\frac{1}{y_{1}} h_{i}^{n}+\chi_{i j}^{D} h_{j}^{m} \\
& Y_{i}^{m D}=b_{\perp}^{D} h_{i}^{m}+\chi_{j i}^{D} h_{j}^{n}
\end{aligned}
$$

with the symmetric material tensor $\chi_{i j}^{D}=\chi_{2}^{D} m_{k} n_{k}\left(\delta_{i j}-n_{i} n_{j}\right)$. The crucial point for its existence is that $\chi_{i j}^{D}$ is odd under $T_{R}$, as well as under the replacement $n_{i} \rightarrow-n_{i}$. For the experiment considered below, the force $h_{i}^{n}$ is due to the Frank rotational elastic energy and a surface anchoring energy, while $h_{i}^{m}$ describes the orienting force of an external field on a ferromagnet; of course, both forces contain contributions from the $A_{2}$ energy given above.

In the studies by Potisk et al. [8, 9], magnetooptic response experiments are described. On a sample of homogeneous structure $n \| m$, a field is applied perpendicularly that rotates the magnetization toward the field direction. Due to the coupling between $m$ and $n$, also the director starts to rotate, which is hindered by the surface anchoring and the subsequent deformation of the director field. Finally, a stationary state is reached that depends on the field strength. Sending light through the sample (along the field direction), using a polarizer and analyzer, the measured phase shift between the ordinary and extraordinary beams allows to monitor the final director orientation, as well as the switching dynamics. It turns out that the director relaxation $\left(\gamma_{1}\right)$ and the magnetization relaxation $\left(b_{\perp}{ }^{D}\right)$ alone are not sufficient to describe the results, but a nonzero $\chi_{2}^{D}$ is mandatory. Indeed, $\chi_{2}^{D}$ turns out to be rather large of the order of $\gamma_{1}$. This is a clear indication that, in such complicated systems, dynamic crosscouplings are important.

There is a reversible counterpart, $\chi_{i j}^{R}=\chi^{R} \epsilon_{i j k} n_{k}$, which is even under $T_{R}$ and leads to a vanishing entropy production due to the antisymmetry of $\epsilon_{i j k}$. The effect of adding those terms to Eqs. (21.26) and (21.27), not yet detected experimentally, would be an out-of plane rotation of the director [9].

Another area to detect the complexity of ferromagnetic nematics is flow. In ordinary nematics, there are basically two flow effects, viscosity and flow alignment due to a reversible coupling between (symmetric) shear flow and director rotations. In 
ferromagnetic nematics, there are additional cross-couplings possible due to the odd $T_{R}$ signature of the magnetization. In particular, there are dissipative couplings between flow and the nematic and magnetic forces, $h_{i}^{n}$ and $h_{i}^{m}$, and a reversible one involving $h_{i}^{m}$ and a reversible version of the viscous coupling [10]. As a result, the velocity profile in a (simple) shear experiment generally deviates from linearity, when a field is present, and effective viscosities become field dependent increasing by a factor up to two in accordance with experiments [46]. In ordinary nematics, there are 3 Miesowicz viscosities according to the three possibilities to fix the director relative to the shear geometry (along the velocity field, along the velocity gradient, and perpendicular to both). In ferromagnetic nematics, there are nine different ways to fix independently (by different external fields) the orientation of $n$ and $m$ relative to shear flow. Due to the various couplings among the variables, these effective viscosities are rather complicated functions of the reversible and irreversible transport parameters involved [10].

\subsubsection{Ferromagnetic nematics with tetrahedral order}

When tetrahedral order is added to ferromagnetic nematics, three preferred structures exist - the nematic director $n_{i}$, the magnetization $m_{i}$, and the tetrahedral structure $T_{i j k}$ (for the latter, refer the study by Fel [25] and the discussion around Eq. (21.9)). The interesting point in this situation is that $m_{i}$ and $T_{i j k}$ have opposite symmetry properties, $\epsilon_{S}=+1 \epsilon_{T}=-1$, and $\epsilon_{S}=-1$, and $\epsilon_{T}=+1$, respectively. We will only consider the case where the director is fixed to be parallel to one of the 2-fold symmetry axes of $T_{i j k}$. This is the structure of $\mathrm{a}_{2 d}$ phase [26]. The hydrodynamic variables are the rotations of the director and the rotation of the tetrahedral structure about the director. Rotations of the magnetization are independent degrees of freedom, but due to an energetic coupling between $n_{i}$ and $m_{i}$, the two directions are parallel in equilibrium, and the phase is uniaxial. For the full hydrodynamics of such a phase, refer the study by Potisk et al. [12].

In the statics, the most prominent feature in the $\mathrm{D}_{2 d}$ phase is the linear gradient term in the energy density $\sim T_{i j k} n_{i} \nabla_{j} n_{k}$. It favors helical structures of $n_{i}$ and $T_{i j k}$ (about a second, perpendicular 2-fold tetrahedral symmetry axis). For the magnetization, there is an analogous linear gradient term, $\sim T_{i j k} m_{i} \nabla_{j} m_{k}$. The sum of the two linear terms favors a combined helical structure of $n_{i}$ and $m_{i}$ (and $T_{i j k}$ ), thereby preserving $n_{i}$ and $m_{i}$ remaining parallel. Since the phase is achiral (there is no pseudoscalar quantity present), this is ambidextrous helicity.

In the reversible dynamics, there are very special couplings that only exist since all three ordered structures are involved. An example is the coupling between the thermal degree of freedom and director rotations, $j_{i}^{\sigma R}=\xi_{i j}^{T n} h_{j}^{n}$ and $Y_{i}^{n R}=\xi_{j i}^{T n} \nabla_{j} T$. Here, $\xi_{j i}^{T n}$ contains linearly $n_{k}, m_{l}$, and $T_{p q r}$, thereby accommodating the $n_{i} \rightarrow-n_{i}$ invariance, ensuring the reversibility of the currents (due to $m_{i}$ ), and compensating for the polarity 
of the heat current and the temperature gradient (due to $T_{p q r}$ ). Since $\xi_{j i}^{T n}$ is symmetric, the two contributions to Eq. (21.17) cancel as required.

In the dissipative dynamics, the magnetization couples, e.g., to the thermal degree of freedom, $j_{i}^{\sigma D}=\psi_{j i}^{T D} h_{j}^{m}$ and $Y_{i}^{m D}=-\psi_{j i}^{T D} \nabla_{j} T$. Here, $\psi_{j i}^{T D}$ contains linearly $m_{l}$ (ensuring the irreversibility of the currents) and $T_{p q r}$ (compensating for the polarity of the heat current and the temperature gradient). Since $\psi_{j i}^{T D}$ is symmetric, the two contributions to Eq. (21.15) are equal and add up as required. They describe that rotations of the magnetization create heat currents, while temperature gradients drive the dynamics of the magnetization.

\subsection{Elasticity with nonnematic order}

In this section, we discuss various elastic systems that show either a polar preferred direction or an ordered tetrahedral structure - finally together with a permanent magnetization. Differences can be traced back to the different symmetry signatures of the order parameters involved.

\subsubsection{Polar and active polar gels}

If in a nematic liquid crystal the director $n_{i}$ is replaced by a polar vector $p_{i}$, the direction of a permanent polarization, a polar (nematic) phase results. Due to the different symmetry properties, $\epsilon_{S}\left(n_{i}\right)=+1$ and $\epsilon_{S}\left(p_{i}\right)=-1$, the polar phase behaves differently compared to the nematic one. In particular, polar phases tend to form splay textures [23] due to a linear splay term $\nabla_{i} p_{i}$ in the energy functional. Neither in nematics nor in ferromagnetic systems linear splay $\left(\nabla_{i} n_{i}\right.$ or $\left.\nabla_{i} m_{i}\right)$ is possible.

In the study by Brand et al. [5], the hydrodynamics of polar gels is given. It turns out that the reversible dynamics is isomorphic to that of nematic gels [47] and need not to be repeated here. In the statics, there is the standard piezoelectric coupling between polarization and strain. Here, in the uniaxial case, it contains three static susceptibilities. There is another static coupling between polar textures and elasticity, $\psi_{i j}^{p} \sim U_{k l}$ and $\Phi_{i j}^{(e l)} \sim \nabla_{k} p_{l}$, with $\psi_{i j}^{p} \equiv(\partial \varepsilon) /\left(\partial \nabla_{i} p_{j}\right)$. Relative rotations between $p_{i}$ and the network, $\Omega_{i} \equiv \delta p_{j} \Omega_{i j}-\delta p_{i}$, couple to bend distortions of the polarization, $L_{i}^{\Omega} \sim \delta_{i k}{ }^{\perp} p_{j} \nabla_{j} p_{k}$ and $\psi_{i j}^{p} \sim \delta_{i k}{ }^{\perp} p_{j} \Omega_{k}$. This effect is not possible in nematic gels (because of the $n_{i} \rightarrow-n_{i}$ invariance) and neither in ferrogels (due to $\epsilon_{\mathcal{T}}\left(m_{i}\right)=-1$ ).

Variants of polar ordered systems are active polar ones, where biological entities (e.g., fish schools, bird flocks, locust swarms, bacteria, etc.) move coherently in a specific direction, without a head to tail symmetry. The preferred direction is given by the velocity, $F_{i}=F f_{i}$, of the active entities. If the movement stops, also the order 
vanishes. To maintain the active velocity, the entities have to provide energy. This energetic intake is dissipated in the system and defines a nonequilibrium state with $F=F_{0}$. Nevertheless, it can be described in the usual hydrodynamic way just adding the driving force [18].

In the study by Pleiner et al. [4], we investigate an active polar, viscoelastic system with a relaxing elasticity, as, e.g., occurs in movements of bacteria colonies in a gel background. The additional variables are the relaxing strain tensor $U_{i j}$, rotations of the preferred direction, $\delta f_{i}$ with $f_{i} \delta f_{i}=0$, relaxation of $F$ (toward $F_{0}$ ), and relative rotations between $f_{i}$ and the network, $\Omega_{i}=f_{j} \Omega_{i j}-\delta f_{i}$. Note that $\Omega_{i}$ has the same symmetry signatures as $f_{i}$. Apart from the active velocity, there is also a passive one, $v_{i}^{(1)}$, describing movements of the passive background. In such a two-fluid system, the question arises, with which velocity a given variable is convected or transported (Eqs. 21.18-21.24). It turned out that there is no general principle to answer this question, and generally, those transport or convection velocities are material dependent, containing passive and active parts $[48,49]$. In a linearized passive dynamics, the transport terms drop out, but in the active case, with a constant active velocity in the stationary state, even the linearized theory contains transport etc. due to the active velocity, and this active transport is an important part of the dynamics. We mention that sometimes a polarization vector is used to describe active transport [50], but due to the different $T_{R}$ signature of the polarization compared to a velocity, the transport becomes "irreversible" violating thermodynamics and opening up an unphysical dissipation channel [4].

Results involving the active polar order and the elasticity are coupled relaxations of compressional strains, $U_{z z}$, with $F$ and shear strains $U_{x z}$ with relative rotations $\Omega_{x}$, where $F$ relaxes to $F_{0}$ and the other quantities to zero. The sound spectrum of a dynamical system is another important aspect of the physics involved. In simple fluids, one has (ordinary) sound with $\omega^{2}=c_{1}^{2} k^{2}$, with $\omega$ and $k$ being the frequency and wave number of the linear excitation, respectively. It is purely reversible, and dissipation only enters at higher $k$-orders. If in addition the active velocity is taken into account, a second sound excitation arises that is coupled to the first one. However, the full sound spectrum is generally no longer invariant under $\omega \rightarrow-\omega$, indicating a nonequilibrium situation [18]: In the stationary state, wave propagation parallel or antiparallel to the active velocity is different. The elastic degree of freedom gives, by coupling to flow, another sound-like excitation (shear elastic wave) that couples to the other sound-like excitations. However, in the present case, elasticity is relaxing, and dissipation does not come at higher $k$ powers but is already present to order $k^{0}$, which makes the final sound spectrum for this material very complicated [4].

\subsubsection{Tetrahedral and ferromagnetic tetrahedral gels}

In the study by Brand and Pleiner [6], we consider tetrahedral gels and elastomers, i.e. elastic media with a tetrahedral ordered structure described by the rank-3 tensor $T_{i j k}$ 
(already discussed above). A liquid phase that only contains tetrahedral order, $\mathrm{T}_{d}$, is optically isotropic since the rank-2 dielectric tensor cannot couple to $T_{i j k}$. When combined with (isotropic) elasticity, a $\varepsilon \mathrm{T}_{d}$ phase arises. Although the time reversal and space inversion properties of polarization and tetrahedral order are the same, the properties of $\varepsilon \mathrm{T}_{d}$ are quite different from the polar (nematic) phase. One reason is that $T_{i i k}=0$ and $\varepsilon \mathrm{T}_{d}$ is apolar. On the other hand, the rank-3 properties of $T_{i j k}$ allow for specific couplings to the elastic tensors, in particular, $j_{i}^{\sigma D} \sim T_{i j k} \Phi_{j k}^{(e l)}$ and $X_{i j}^{(e l) D} \sim T_{i j k} \nabla_{k} T$ describe a dissipative coupling between elasticity and the thermal degree of freedom. The specific aspect of this coupling lies in the geometry - elastic shear stresses induce a heat current perpendicular to the shear plane, and a temperature gradient induces growth of perpendicular elastic shear strains. For a relaxing elasticity, this results finally in a stationary induced strain. Out-ofplane phenomena are characteristic for tetrahedral order.

If chirality is present (due to a pseudoscalar $q_{0}$ ), a T phase occurs in the liquid case [28] and a $\varepsilon \mathrm{T}$ in the elastic case. The combination $q_{0} T_{i j k}$ gives a $S_{I}$-positive rank-3 tensor that allows, particularly, couplings of the elastic degree of freedom with rotations of the tetrahedral structure, $\delta \Gamma_{i}$, and with relative rotations, $\Omega_{i}$, (in the statics and in the dissipative dynamics) and with flow (in the reversible dynamics). Here, $\Omega_{i}=\epsilon_{i j k} \Omega_{j k}-\delta \Gamma_{i}$ means rotations of the tetrahedral orientation with respect to the elastic medium.

If a tetrahedral gel or elastomer is in addition ferromagnetic, the different time reversal and space inversion signatures of the tetrahedral structure and the permanent magnetization allow for a very rich hydrodynamic theory [13]. We assume the magnetization to be rigidly connected to one of the 2-fold symmetry axes of the tetrahedral structure. This preferred direction is taken as the $z$-direction. Relative rotations here mean combined rotations of $m_{i}$ and $T_{i j k}$ relative to the elastic network.

We concentrate on the interplay of elasticity and magnetization in the presence of tetrahedral order. First, shear strains create (statically) magnetization patterns $\nabla_{z} m_{x} \sim U_{y z}$ and $\nabla_{z} m_{y} \sim U_{x z}$ with the magnetization perpendicular to the shear plane. Compressional strains lead to magnetization patterns in the perpendicular plane $\left(\nabla_{x} m_{y}+\nabla_{y} m_{x}\right) \sim U_{z z}$. This should not be mixed up with the standard magnetostriction, where compressional strains $U_{z z}$ or $U_{x x}+U_{y y}$ result in changes of the magnitude of the magnetization, $\delta M / M_{0}$. In addition, there is an indirect coupling of temperature gradients to elastic shear stresses, mediated by relative rotations, such that $\nabla_{x} T$ and $\nabla_{y} T$ create $\Phi_{y z}^{(e l)}$ and $\Phi_{x z}^{(e l)}$, respectively.

In the case of a transient network, a temperature gradient along the preferred axis triggers temporal changes of the strains, which finally result in a stationary elastic shear stress, $\Phi_{x y}^{(e l)}$, in the perpendicular plane. This is due to a reversible as well as an irreversible coupling. In addition, there are also planar compressional stationary strains $\left(\Phi_{y y}^{(e l)}=-\Phi_{x x}^{(e l)}\right)$ due to reversible couplings.

The transverse directions $x$ and $y$ introduced in the previous paragraphs are not determined without a suitable external force. The system is uniaxial and transversely 
isotropic, optically. Of course, the tetrahedral structure has a fourfold (improper) symmetry, only in optical measurements this cannot be detected. However, if one applies the external temperature gradient perpendicularly (e.g., $\nabla_{x} T$ ), the transverse isotropy is broken, which can be detected optically. Measuring the ratio $\Phi_{y z}^{(e l)} / \Phi_{x z}^{(e l)}$ of the induced stationary elastic strains, one gets the orientation of the transverse tetrahedral structure relative to the externally defined $x$-direction [13].

\subsection{Magnetorheological fluids}

\subsubsection{A minimal hydrodynamic model}

In the study by Potisk et al. [14], we discuss a simple, minimal model to describe the static and dynamic properties of magnetorheological fluids (MRFs). The main ingredient is transient elasticity (relaxing strain variable) that is induced by an external magnetic field. We model this by assuming the elastic moduli and the relaxation times to be proportional to $M^{2}$, where $M_{i}$ is the magnetization, which is zero in equilibrium and in the absence of an external field. Obviously, this description can only be applied to small and intermediate fields, since for high fields there are saturation effects and the elastic moduli will not grow indefinitely. Important is also magnetostriction that provides a static coupling between elasticity and magnetization. In order to make this magnetorheological model as simple as possible, thermal and concentration effects have been neglected, e.g., disregarding temperature gradients and sedimentation. Isotropy of the material properties is assumed, thereby also eliminating relative rotations as variables.

From the symmetry point of view, this model is like a magnetic, viscoelastic suspension. The most important aspects are not due to symmetry reasons but come from internal material properties, like the tendency of the magnetic particles to form chains (and induce elasticity) in the presence of external fields. Another example for a special internal material property is the field dependence of the viscosity in some magnetic fluids [51]. The influence of this property on the threshold behavior in thermal instabilities has been investigated theoretically in the study by Pérez et al. [7].

The material is assumed to be within two parallel plates with the magnetic field $H_{i}=\delta_{i z} H_{0}$ perpendicular to them. First, we have applied this magnetorheological model to external static shear deformations (strains) $S_{i j}=S \delta_{i z} \delta_{j x}$. The two forces applied lead to three coupled nonlinear equations for the elastic shear stress, $\Psi_{x z}$, the magnetization parallel to the field, $M_{z}$, and the component $M_{x}$ perpendicular to the external field (compare Eqs. 21.29-21.31 in ref.[14]). Analysis of these equations shows that all three quantities are nonvanishing as a consequence of the magnetostrictive coupling (in this geometry and with two external forces) of $M_{x}, M_{z}$, and $\Psi_{x z}$ in the energy, as well as in the resulting minimizing equations. The resulting elastic shear stress, $\Psi_{x z}$, is proportional to $H_{0}^{2}$ and initially grows linearly as a function of $S$, then reaches a maximum (the 
"static yield stress") at a field-independent yield strain, and diminishes beyond. This is the unstable region, where elasticity breaks down and gives way to a liquid-like response. The tilt of the magnetization, characterized by a nonzero $M_{x}$, increases with $S$. If a normal pressure is applied additionally, the system becomes more rigid, and the static yield stress (as well as the appropriate yield strain) increases considerably. These effects are qualitatively in accordance with experiments.

Under external shear flow, $v_{x}=\dot{y} z$ with shear rate $\dot{\gamma}$, one has to consider the full dynamic equations for the magnetization, the elasticity, and flow. The most important cross-coupling is between the magnetization and flow. It comes in two parts, the first is the convection term $\epsilon_{i j k} M_{j} \omega_{k}$ for $\dot{M}_{i}$ in Eq. (21.23) and the second is a phenomenological contribution to the magnetization current $Y_{i}^{M R} \sim c_{i j k}^{R} A_{j k}$. Both contributions are reversible since $c_{i j k}^{R}$ contains an odd number of $M_{i}$ factors. The shear part of the latter, $c_{2}^{R}$, is the analog to the flow alignment parameter in nematic liquid crystals and has been given in the context of ferrofluids in the studies by Müller and Liu [52] and Müller et al. [53]. The counter terms, necessary to guarantee zero entropy production, then enter the full stress tensor, $\sigma_{i j}$, in addition to the viscous stress due to the external flow and the elastic stress.

For stationary shear flow, where $\dot{\gamma}=\dot{\gamma}_{0}$ is a constant, the full stress tensor shows, as a function of $\dot{y}_{0}$, a steep increase up to, what is called the dynamic yield shear stress, beyond which the increase is much slower. The dynamic yield shear stress increases quadratically with the external field $H_{0}$. The behavior at higher $\dot{\gamma}_{0}$ shows shear thinning and therefore fits better to a Casson or Herschel-Bulkley model [54], rather than to the standard Bingham model.

For oscillating shear flow, $\dot{\gamma}=\dot{y}_{0} \cos \omega t$, the linear response of the system is described by a complex shear modulus, $\mathrm{G}=\mathrm{G}^{\prime}+\mathrm{iG}^{\prime \prime}$, whose real and imaginary parts describe the reactive (reversible) and dissipative response, respectively. As a function of the frequency, the storage modulus $G^{\prime}$ increases quadratically until it reaches a constant plateau value. The loss modulus $G^{\prime \prime}$ increases linearly up to maximum and decreases until a minimum is reached and finally grows linearly again according to a simple viscous behavior. These features correspond fairly well to experimental findings $[55,56]$. The shear modulus $|G|$ can exhibit two plateaus, at low and at intermediate frequencies, related to the two relaxation processes involved, strain relaxation and magnetic relaxation. The Cox-Merz rule, an empirical law relating nonlinear stationary to linear high frequency properties [57, 58], is often fulfilled in polymer dynamics but not in our model of MRFs. The difference is probably due to the fact that the columnar structure is destroyed under large steady shear but not when small amplitude oscillatory shear is applied.

\subsubsection{A two-fluid description}

In the minimal model of MRFs, we have used only one velocity field to describe the dynamics of all variables. Similar to the case of suspensions of large particles or in the 
dynamics of phase separation, we allow in a two-fluid description [15] macroscopic movements of the magnetic particles relative to the background fluid. Therefore, we introduce two density variables, $\rho_{1}$ and $\rho_{2}$ (as in a binary mixture), and two momenta $g_{i}^{(1)}=\rho_{1} v_{i}^{(1)}$ and $g_{i}^{(2)}=\rho_{2} v_{i}^{(2)}$. Compared to the minimal model, there are now two new variables, the concentration $\phi=\rho_{2} / \rho$ and the relative velocity $w_{i}=v_{i}^{(1)}-v_{i}^{(2)}$ that come together with the conserved variables, total density $\rho=\rho_{1}+\rho_{2}$ and total momentum $g_{i}=g_{i}^{(1)}+g_{i}^{(2)}$. The concentration is even under $T_{R}$ and $S_{I}$, while the relative velocity is odd under both.

This two-fluid description is different from the case of active polar gels in Section 21.3.1, where the second velocity is related to a broken symmetry and hence a class B variable. Here, the relative velocity $w_{i}$ is simply a slowly relaxing variable of class $\mathrm{C}$ with the conjugate $h_{i}^{w}\left(\mu_{C} d C \rightarrow h_{i}^{w} d w_{i}\right)$ and the balance equation $(\partial / \partial t) w_{i}+Y_{i}^{w}=0$. The conjugate quantity to $\phi$ is the osmotic pressure $\Pi$ and the (linearized) dynamic equation reads $\rho_{0}(\partial / \partial t) \phi+\nabla_{i} j_{i}^{\phi}=0$ (for the general case, refer the study by Pleiner et al. [15]). The conjugates $h_{i}^{w}$ and have the same symmetry signatures as $w_{i}$ and $\phi$, respectively. The currents $j_{i}^{\phi}$ and $Y_{i}^{w}$ are odd under $S_{I}$, while the second law of thermodynamics requires $j_{i}^{\phi R}$ and $Y_{i}^{w D}$ to be odd under $T_{R}$ and $j_{i}^{\phi D}$ and $Y_{i}^{w R}$ to be even.

The general problem of any two-fluid theory regarding the form of the transport velocities, e.g., how to generalize Eqs. 21.18-21.24, cannot be discussed here but refer the studies by Pleiner and Harden [48, 49]. However, as part of our two-fluid model of MRFs, we choose velocity $v_{i}^{(1)}$ to transport or convect the variables $\rho_{1}$ and $g_{i}^{(1)}$, while for the magnetization $M_{i}$ and the strain $U_{i j}$, we take $v_{i}^{(2)}$ (and heat is transported by the mean velocity $v_{i}=g_{i} / \rho$ ). In Pleiner et al. [15], we show how this choice fits well into the general scheme without violating any thermodynamic rule, if one requires $v_{i}^{(c)}=$ $\left(\rho_{1} / \rho\right) v_{i}^{(2)}+\left(\rho_{2} / \rho\right) v_{i}^{(1)}$ to be the transport velocity for $\phi$ and $w_{i}$.

In the statics, $w_{i} \sim h_{i}^{w}$ and does not couple to other degrees of freedom (in a linearized description), while the concentration couples to the magnetization and the (trace of the) strain, $\delta \Pi \sim\left(2 \chi_{m} M_{i}^{0} \delta M_{i}+\tilde{\chi}_{u} \delta U_{k k}\right)$, and the counter terms (to make sure $\varepsilon$ is a potential) are $h_{i}^{M}=2 \chi_{m} M_{i}^{0} \delta \phi$ and $\Phi_{i j}=2 \tilde{\chi}_{u} \delta_{i j} \delta \phi$, with $\tilde{\chi}_{u}=M_{0}^{2} \chi_{u}$ in accordance with the similar form of the elastic moduli.

In the dynamics, the relative velocity reversibly couples to the magnetization, $Y_{i}^{w R}=\xi_{i j k} \nabla_{j} h_{k}^{M}$ and $X_{i}^{M R}=\xi_{k j i} \nabla_{j} h_{k}^{w}$, with $\xi_{i j k}$ containing only odd powers of $M_{i}$, as well as irreversibly, $Y_{i}^{w D}=-\nabla_{j}\left(\gamma_{j i k} h_{k}^{M}\right)$ and $X_{i}^{M D}=\gamma_{k j i} \nabla_{j} h_{k}^{w}$, where $\gamma_{i j k}$ is even in $M_{i}$. The relative velocity also couples to flow $Y_{i}^{w D}=-\nabla_{j}\left(v_{j i k l}^{(c)} A_{k l}\right)$ and $\sigma_{i j}^{D}=-v_{i j k l}^{(c)} \nabla_{l} h_{k}^{w}$, where $v_{i j k l}^{(c)}$ is even in $M_{i}$. The concentration couples to magnetization, $j_{i}^{\phi D}=-\alpha_{k i j} \nabla_{j} h_{k}^{M}$ and $Y_{i}^{M D}=-\nabla_{k}\left(\alpha_{i j k} \nabla_{j} \Pi\right)$, where $\alpha_{i j k}$ is odd in $M_{i}$ in order to be dissipative.

A direct comparison with the one-fluid model can be made for a stationary shear flow in the parallel plate geometry. Assuming as boundary conditions at the moving 
plate for the background flow, $v_{x}^{(1)}=\Gamma_{x}$, for the particle phase, $v_{i}^{(2)}=0$, and for the strain, $\nabla_{z} U_{x z}=0$, one finds a nonzero and asymmetric relative velocity field $w_{x}$, whose maximum depends on the external shear strain rate $\Gamma_{x}$ in a complicated way. As a result, the flow profile (of $v_{x}^{(1)}$ ) in the flow channel is not linear (in contrast to the onefluid case). In addition, the stress-strain relation, although quite similar to the one-fluid case for small and large $\Gamma_{x}$, differs for intermediate $\Gamma_{x}$ values, in particular, the somewhat unphysical overshoot present in the one-fluid case is no longer there. The introduction of a two-fluid description considerably improves the quality of the macroscopic model.

Bridging the gap between MRFs and soft magnetorheological gels, we would like to refer to the recent review of the understanding of the interplay between single particle motion, internal deformation, and matrix properties, in particular, concerning the buckling of chains and the matrix deformations around inclusions [59].

\subsection{Summary and perspective}

In this compact review, we have first outlined the approach of macroscopic dynamics based on the use of linear irreversible thermodynamics and the behavior under symmetry operations including inversion, time reversal, and rotations. The variables of interest come in three groups: conservation laws, variables associated with spontaneously broken continuous symmetries, and macroscopic variables, which relax on a sufficiently long time scale to be of hydrodynamic interest.

One field covered is the interaction of nematic order with magnetism. It includes the description of isotropic and uniaxial magnetic elastomers and gels, as well as ferromagnetic nematics, as they have become available experimentally a few years ago. Stimulated by this progress, we also investigated the effect of additional tetrahedral/octupolar order. Another related topic is the study of elasticity with nonnematic order including polar and active polar gels, as well as tetrahedral and ferromagnetic tetrahedral gels.

To examine the effect of larger particles in a carrier fluid, we have presented a minimal one-fluid model of MRFs, the results of which are in accord with a large body of experimental literature. To account for segregation effects between the carrier fluid and the particles, we generalized our approach very recently and gave a two-fluid description of MRFs.

As a perspective, we just mention two classes of systems for which the presented approach will be useful. One is the field of active magnetic gels and elastomers. Quite recently, it has become clear that magnetotactic bacteria fall into this class of systems. Another direction for the near future is the study of fluid- or gel-like systems, which show simultaneously magnetic and electric order. We are thinking, for example, of a system, which is ferroelectric and ferromagnetic simultaneously. Candidates are the 
ferromagnetic nematic liquid crystals already existing with a solvent, which contains ferroelectric particles. One might thus obtain a liquid multiferroic system with three types of order: ferromagnetic, ferroelectric, and nematic.

Acknowledgment: The authors thank the Deutsche Forschungsgemeinschaft for partial support over the last few years via the SPP 1681. The authors take pleasure to thank Tilen Potisk and Daniel Svenšek for the fruitful collaboration within the SPP1681 and Stefan Odenbach for initiating and coordinating this stimulating program.

Author contribution: All the authors have accepted responsibility for the entire content of this submitted manuscript and approved submission.

Research funding: This research was supported by the Deutsche Forschungsgemeinschaft through SPP1681.

Conflict of interest statement: The authors declare no conflicts of interest regarding this article.

\section{References}

1. Brand HR, Pleiner H, Svenšek D. Reversible and dissipative macroscopic contributions to the stress tensor: active or passive?. Eur Phys J E 2014;37:83.

2. Brand HR, Pleiner H. Macroscopic behavior of ferronematic gels and elastomers. Eur Phys J E 2014; 37:122.

3. Brand HR, Fink A, Pleiner H. Macroscopic behavior of ferrocholesteric liquid crystals and ferrocholesteric gels and elastomers. Eur Phys J E 2015;38:65.

4. Pleiner H, Svenšek D, Brand HR. Hydrodynamics of active polar systems in a (Visco)elastic background. Rheol Acta 2016;55:857.

5. Brand HR, Pleiner H, Svenšek D. Macroscopic behavior of polar nematic gels and elastomers. Eur Phys J E 2016;39:105.

6. Brand HR, Pleiner H. On the influence of a network on optically isotropic fluid phases with tetrahedral/octupolar order. Eur Phys J E 2017;40:34.

7. Pérez LM, Bragard J, Díaz P, Mancini HL, Laroze D, Pleiner H. Magneto-viscous effect on thermal convection thresholds in an Oldroyd magnetic fluid. J Magn Magn Mater 2017;444:432.

8. Potisk T, Svenšek D, Brand HR, Pleiner H, Lisjak D, Ostermann N, et al. Dynamic magneto-optic coupling in a ferromagnetic nematic liquid crystal. Phys Rev Lett. 2017;119:097802.

9. Potisk T, Mertelj A, Sebastián N, Ostermann N, Lisjak D, Brand HR, et al. Magneto-optic dynamics in a ferromagnetic nematic liquid crystal. Phys Rev E 2018;97:012701.

10. Potisk T, Svenšek D, Pleiner H, Brand HR. Effects of flow on the dynamics of a ferromagnetic nematic liquid crystal. Phys Rev E 2018;97:042705.

11. Brand HR, Pleiner $H$, Svenšek D. Dissipative versus reversible contributions to macroscopic dynamics: the role of time-reversal symmetry and entropy production. Rheol Acta 2018;57:773.

12. Potisk T, Pleiner H, Brand HR. Dynamic interplay of nematic, magnetic, and tetrahedral order in ferromagnetic nematic phases. Phys Rev E 2018;98:042703.

13. Potisk T, Pleiner H, Brand HR. Influence of tetrahedral order on ferromagnetic gel phases. Eur Phys J E 2019;42:35.

14. Potisk T, Svenšek D, Pleiner H, Brand HR. Continuum model of magnetic field induced viscoelasticity in magnetorheological fluids. J Chem Phys 2019;150:174901. 
15. Pleiner H, Svenšek D, Potisk T, Brand HR. Macroscopic two-fluid effects in magnetorheological fluids. Phys Rev E 2020;101:032601.

16. Martin PC, Parodi O, Pershan PS. Unified hydrodynamic theory for crystals, liquid crystals, and normal fluids. Phys Rev A 1972;6:2401.

17. Pleiner $\mathrm{H}, \mathrm{Liu} M, \mathrm{~B}$, Band HR. The structure of convective nonlinearities in polymer rheology. Rheol Acta 2000;39:560.

18. Pleiner H, Svenšek D, Brand HR. Active polar two-fluid macroscopic dynamics. Eur Phys J E 2013;36: 135.

19. Menzel A, Pleiner $\mathrm{H}$, Brand $\mathrm{HR}$. Nonlinear relative rotations in liquid crystalline elastomers. J Chem Phys 2007;126:234901.

20. Brand HR, Pleiner H. New theoretical results for the Lehmann effect in cholesteric liquid crystals. Phys Rev A 1988;37:2736.

21. Cladis PE, Brand HR, Pleiner H. Macroscopic properties of smectic C_G liquid crystals. Eur Phys J B 1998;6:347.

22. Cladis PE, Brand HR, Pleiner H. Fluid biaxial banana smectics: symmetry at work. Liq Cryst Today 1999;9:1.

23. Pleiner H, Brand HR. Spontaneous splay phases in polar nematic liquid crystals. Europhys Lett 1989;9:243.

24. Brand HR, Pleiner H, Ziebert F. Macroscopic dynamics of polar nematic liquid crystals. Phys Rev $E$ 2006;74:021713.

25. Fel LG. Tetrahedral symmetry in nematic liquid crystals. Phys Rev E 1995;52:702.

26. Brand HR, Pleiner H. Macroscopic behavior of non-polar tetrahedratic nematic liquid crystals. Eur Phys J E 2010;31:37.

27. Pleiner $\mathrm{H}$, Brand $\mathrm{HR}$. Low symmetry tetrahedral nematic liquid crystal phases: ambidextrous chirality and ambidextrous helicity. Eur Phys J E 2014;37:11.

28. Pleiner H, Brand HR. Tetrahedral order in liquid crystals. Braz J Phys 2016;46:565.

29. Pleiner H, Brand HR. Hydrodynamics and electrohydrodynmaics of liquid crystals. In: Buka A, Kramer L, editors. Pattern formation in liquid crystals. New York: Springer; 1996;15-67 pp.

30. Leslie FM. Some thermal effects in cholesteric liquid crystals. Proc Roy Soc A 1968;307:359.

31. Brand HR, Pleiner H, Cladis PE. Flow properties of the optically isotropic tetrahedratic phase. Eur Phys J E 2002;7:163.

32. Jarkova E, Pleiner H, Müller H-W, Brand HR. Hydrodynamics of isotropic ferrogels. Phys Rev E 2003; 68:041706.

33. Attaran A, Brummund J, Wallmersperger T. Development of a continuum model for ferrogels. J Intell Mater Syst Struct 2017;28:1358.

34. Weeber R, Kreissl P, Holm C. Studying the field-controlled change of shape and elasticity of magnetic gels using particle-based simulations. Arch Appl Mech 2019;89:3.

35. Menzel AM. Mesoscopic characterization of magnetoelastic hybrid materials: magnetic gels and elastomers, their particle-scale description, and scale-bridging links. Arch Appl Mech 2019;89:17.

36. Pessot G, Schümann M, Gundermann T, Odenbach S, Löwen H, Menzel AM. Tunable dynamic moduli of magnetic elastomers: from characterization by X-ray micro-computed tomography to mesoscopic modeling. J Phys Condens Matter 2018;30:125101.

37. Becker TI, Böhm V, Chaves Vega J, Odenbach S, Raikher YL, Zimmermann K. Magnetic-fieldcontrolled mechanical behavior of magneto-sensitive elastomers in applications for actuator and sensor systems. Arch Appl Mech 2019;89:133.

38. Collin D, Auernhammer GK, Gavat O, Martinoty P, Brand HR. Frozen-in magnetic order in uniaxial magnetic gels: preparation and physical properties. Macromol Rapid Commun 2003;24:737.

39. Bohlius S, Brand HR, Pleiner H. Macroscopic dynamics of uniaxial magnetic gels. Phys Rev E 2004; 70:061411. 
40. Menzel AM. Bridging from particle to macroscopic scales in uniaxial magnetic gels. J Chem Phys 2014;141:194907.

41. Nadasi H, Corradi A, Stannarius R, Koch K, Schmidt AM, Aya S, et al. The role of structural anisotropy in the magnetooptical response of an organoferrogel with mobile magnetic nanoparticles. Soft Matter 2019;15:3788.

42. Mertelj A, Lisjak D, Drofenik M, Cǒpič M. Ferromagnetism in suspensions of magnetic platelets in liquid crystal. Nature 2013;504:237.

43. Mertelj A, Osterman N, Lisjak D, Cǒpič M. Magneto-optic and converse magnetoelectric effects in a ferromagnetic liquid crystal. Soft Matter 2014;10:9065.

44. Jarkova E, Pleiner H, Müller H-W, Brand HR. Macroscopic dynamics of ferronematics. J Chem Phys 2003;118:2422.

45. Shrivastav GP, Klapp SHL. Anomalous transport of magnetic colloids in a liquid crystal-magnetic colloid mixture. Soft Matter 2019;15:973.

46. Sahoo R, Rasna MV, Lisjak D, Mertelj A, Dahra S. Magnetodielectric and magnetoviscosity response of a ferromagnetic liquid crystal at low magnetic fields. Appl Phys Lett 2015; 106: 161905.

47. Brand HR, Pleiner $H$. Electrohydrodynamics of nematic liquid crystalline elastomers. Physica $A$ 1994;208:359.

48. Pleiner H, Harden JL. In Nonlinear problems of continuum mechanics, special issue of notices of universities, South of Russia. Nat Sci 2003;46. arXiv:cond-mat/0404134.

49. Pleiner H, Harden JL. General nonlinear 2-fluid hydrodynamics of complex fluids and soft matter. AIP Proc 2004;708:46.

50. Marchetti MC, Joanny JF, Ramaswamy S, Liverpool TB, Prost J, Rao M, et al. Hydrodynamics of soft active matter. Rev Mod Phys 2013;85:1143.

51. Odenbach S. Magnetoviscous effects in ferrofluids Lecture Notes in Physics Monographs. Berlin: Springer; 2002.

52. Müller H-W, Liu M. Structure of ferrofluid dynamics. Phys Rev E 2001;64:061405.

53. Müller $\mathrm{O}$, Hahn D, Liu M. Non-newtonian behaviour in ferrofluids and magnetization relaxation. J Phys Condens Matter 2006;18:S2623.

54. Susan-Resiga D. A rheological model for magnetorheological fluids. J Intell Mater Syst Struct 2009; 20:1001.

55. Lee JH, Lu Q, Lee JY, Choi HJ. Polymer-magnetic composite particles of $\mathrm{Fe}_{3} \mathrm{O}_{4} /$ Poly(o-anisidine) and their suspension characteristics under applied magnetic fields. Polymers 2019;11:219.

56. Arief I, Mukhopadhyay PK. Magnetorheological Payne effect in bidisperse MR fluids containing Fe nanorods and $\mathrm{Fe}_{3} \mathrm{O}_{4}$ nanospheres: A dynamic rheological study. J Alloys Compd 2017;696:1053.

57. Cox WP, Merz EH. Correlation of dynamic and steady flow viscosities. J Polym Sci 1958;28:619.

58. Müller O, Liu M, Pleiner H, Brand HR. Transient elasticity and polymeric fluids: Small-amplitude deformations. Phys Rev E 2016;93:023114.

59. Auernhammer GK. Magnetorheological gels in two and three dimensions: understanding the interplay between single particle motion, internal deformations, and matrix properties. Arch Appl Mech 2019;89:153. 
